\title{
Mutation profile in Indian primary myelofibrosis patients and its clinical implications
}

\author{
Vinod R. Patil, S. Chandrakala, Shruti Mantri, Rajesh Patil, Nilesh Wasekar, Farah Jijina
}

\begin{abstract}
Background: Primary myelofibrosis (PMF) is a myeloproliferative neoplasm (MPN) characterized by abnormal proliferation of megakaryocytes, bone marrow fibrosis, and extramedullary hematopoiesis. We did mutation profile of 50 patients of PMF and tried to correlate it with initial clinical presentation of these patients. Materials and Methods: All new and follow up patients who were diagnosed as PMF based on WHO 2016 definition of PMF were included. Mutation profile of these patients including JAK2 V6I7F, JAK2 exon I2, CALR and MPL mutations was done and all clinical, demographic and laboratory details were recorded. Results: Total 50 patients were enrolled out of which 29 were males and 21 were females. Out of these patients, 32 (64\%) were JAK2 positive, 13 (26\%) were CALR positive, I (2\%) were MPL positive and 4 ( $8 \%$ ) were triple negative. As compared to JAK2+ve patients and triple negative group, CALR positive patients were younger, had lower total leucocyte count, larger spleen size, lower dynamic international prognostic scoring system (DIPSS) score and higher grade of fibrosis of marrow. Conclusion: This study depicts that incidence of JAK2 and CALR mutations in Indian PMF patients is fairly similar to that in rest of the world. CALR positive patients have better clinical parameters at presentation and have better prognosis as compared to JAK2 positive patients.
\end{abstract}

Key words: Indian, mutations, myelofibrosis

\section{Introduction}

Primary myelofibrosis (PMF) is a type of myeloproliferative neoplasm that is characterized by fibrosis of bone marrow, abnormal proliferation of atypical megakaryocytes, symptoms due to circulating cytokines, and extramedullary hematopoiesis. ${ }^{[1]}$ Although the pathogenesis of PMF is not fully understood, the genetic basis of disease began to unravel with the discovery of Janus kinase 2 (JAK2) mutations in 2005. ${ }^{[2]}$ Subsequently, myeloproliferative leukemia protein (MPL) and JAK2 exon 12 mutations were discovered, and only recently in 2013, two groups independently discovered the calreticulin (CALR) mutations in PMF population with unmutated JAK2 and MPL genes. ${ }^{[3,4]}$ As against polycythemia vera, genetic mutations in PMF are heterogeneous. ${ }^{[1]}$ Thus, many studies are being done to assess the clinical implications of these numerous mutations in the course of disease and its prognostication. Very few such studies are done in India until now. We hereby present data of 50 PMF patients with their mutation profile and correlation with the clinical parameters.

\section{Materials and Methods}

This prospective observational study was conducted in Dr. J. C. Patel Department of Clinical Hematology in KEM Hospital, Mumbai. All new and follow-up patients who were diagnosed as PMF based on the WHO 2016 definition of PMF were included in the study. Mutation profile of these patients was done, and all clinical and demographic details were recorded. JAK2 V617F, JAK2 exon 12, CALR, and MPL mutations were done in all patients. JAK2 mutation study was conducted using genomic DNA-based polymerase chain reaction (PCR) in combination with fluorescence-based capillary electrophoresis. CALR mutation detection was carried out by PCR - Sanger DNA sequencing using ABI3130XL DNA sequencer. MPL mutation was carried out by Sanger DNA sequencing technique

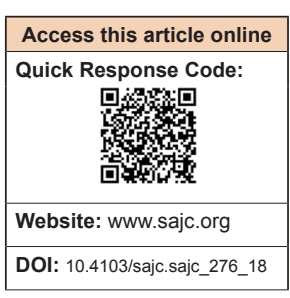

Superspeciality Medical Officer, Department of Clinical Hematology, Seth G. S. Medical College and KEM Hospital, Parel, Mumbai, Maharashtra, India Correspondence to: Dr.Vinod R. Patil, E-mail: dr.vrp85@gmail.com using fluorescent-based capillary electrophoresis on ABI 3130XL DNA sequencer.

As only one patient was MPL positive, patients were grouped as JAK2 positive (V617F or exon12), CALR positive, and triple negative (i.e., negative for all three mutations). Clinical and hematological parameters were compared in these groups using paired $t$-test. A value of $P<0.05$ was considered statistically significant.

\section{Results}

A total of 50 patients were enrolled of which 29 were male and 21 were female. Their clinical symptoms or signs at presentation are depicted as bar diagram in Figure 1. The most common symptom of the presentation was pain/discomfort due to lump in the abdomen (34/50) followed by fatigue or weakness $(25 / 50)$.

Of these patients, 32 (64\%) were JAK2 positive, $13(26 \%)$ were CALR positive, $1(2 \%)$ were MPL positive, and $4(8 \%)$ were triple negative [Figure 2]. CALR mutation was seen in $72 \%$ of JAK2-unmutated patients. None of the patients was positive for JAK2 exon 12 mutation.

Clinical parameters such as age at presentation, total leukocyte count, spleen size, dynamic international prognostic scoring system (DIPSS) score, and grade of marrow fibrosis showed the statistically significant difference. As compared to JAK2-positive patients and triple-negative group, CALR-positive patients were younger and had lower total leukocyte count, larger spleen size, lower DIPSS score, and higher grade of fibrosis of marrow. Other blood counts and duration of symptoms were not statistically different in these patients. Although thrombosis was seen in three patients of JAK2-mutated group and none in other groups, it failed to show statistical significance. The average DIPSS score was higher in triple-negative patients. These findings are depicted in Table 1.

This is an open access journal, and articles are distributed under the terms of the Creative Commons Attribution-NonCommercial-ShareAlike 4.0 License, which allows others to remix, tweak, and build upon the work non-commercially, as long as appropriate credit is given and the new creations are licensed under the identical terms.

For reprints contact: reprints@ @medknow.com

How to cite this article: Patil VR, Chandrakala S, Mantri S, Patil R, Wasekar N, Jijina F. Mutation profile in Indian primary myelofibrosis patients and its clinical implications. South Asian J Cancer 2019;8: 186-8. 


\section{Discussion}

PMF is a type of myeloproliferative neoplasm with heterogeneous mutations. Although JAK2 is the most common, CALR mutation is also being increasingly detected. Discovery of CALR mutations has set off many studies to associate these and other mutations with clinical and prognostic implication in PMF patients. The incidence of CALR in our patients is $26 \%$ overall and $72 \%$ of JAK2-unmutated patients. This is the same as many western studies as shown in Table 2. One of the patients had MPL mutation.

Another similar study done in India previously by the All India Institutes of Medical Sciences showed lower incidence of CALR mutation as compared to our and other studies. They included 80 patients of which 45 (56.2\%), 9 (11.2\%), and $26(32.6 \%)$ were JAK2 positive, CALR positive, and triple negative, respectively. This may indicate regional variation in CALR mutation incidence in our country. However, larger nationwide studies are necessary for proving this hypothesis.

Of all the CALR exon 9 mutated patients, 3 patients had 52 bp (type 1) mutation, 1 had 50 bp mutation, 1 had $30 \mathrm{bp}$ mutation, and 8 patients had 51 bp mutation. When seen clinically, CALR-mutated patients were younger as compared to JAK2 and triple-negative patients. This is the same as studies by Rumi et al., ${ }^{[7]}$ Sazawal et al., ${ }^{[8]}$ and Wojtaszewska ${ }^{[9]}$ et al. although some studies like Guo

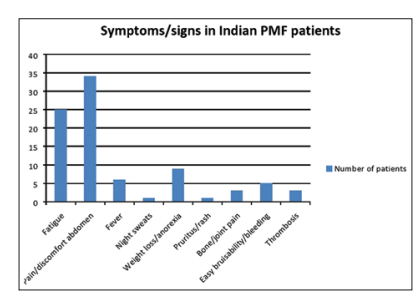

Figure 1: Clinical features of patients of myelofibrosis

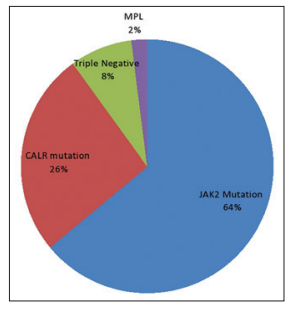

Figure 2: Distribution of mutations in myelofibrosis et $a{ }^{[10]}$ and Pietra ${ }^{[1]}$ et al. showed no age difference. This variation in studies may be attributed to the different ethnic origin of these patients. Although many western studies by Pietra et al,,$^{[1]}$ Rumi et al.,,$^{[7]}$ Tefferi et al., ${ }^{[11]}$ and Wojtaszewska et al. ${ }^{[9]}$ showed that CALR-mutated patients have higher hemoglobin, platelet counts, and lower total leukocyte counts compared to JAK2-mutated patients, we did not get any difference in hematological parameters of these patients. This is in compliance with Sazawal et al. ${ }^{[8]}$ which may suggest that Indian patients of CALR-mutated PMF have no difference in blood counts compared to JAK2-mutated patients. CALR-mutated patients have lower DIPSS score as against JAK2-mutated or triple-negative patients. This may implicate that these patients have better prognosis and overall survival. This result is consistent with Tefferi et al. ${ }^{[11]}$ but not with Sazawal et al., ${ }^{[8]}$ Wojtaszewska et al., ${ }^{[9]}$ Cabagnols et al., ${ }^{[12]}$ and Pietra et al. ${ }^{[1]}$ The spleen size of CALR-positive patients is larger as compared to JAK2-positive patients as is documented in various other studies. ${ }^{[11,12]}$ Higher degree of marrow fibrosis was seen in patients of CALR-mutated PMF in our study. Overall, these findings suggest that hematological parameters in both JAK2- and CALR-positive PMF patients are similar in Indian patients as against western countries. No statistical difference in clinicohematological parameters was seen between JAK2-mutated and triple-negative patients. As all patients received symptom-directed treatment, we could not assess these patients for correlation of mutation profile with treatment response or overall survival rates. As our study ( $2 \%$ MPL mutation incidence), other studies also showed very low incidence of MPL mutation in PMF patients (ranging from $0 \%$ to $6.4 \%$ ). ${ }^{[3,4,5,7]}$

\section{Conclusion}

This study depicts that the incidence of JAK2 and CALR mutations in Indian PMF patients is fairly similar to

Table 1: Clinical parameters in primary myelofibrosis patients as per their genetic mutation

\begin{tabular}{|c|c|c|c|c|c|}
\hline Parameter (all values in average) & $\begin{array}{c}\text { Total } \\
(n=50)\end{array}$ & $\begin{array}{c}\text { JAK2 positive } \\
(n=32)\end{array}$ & $\begin{array}{c}\text { CALR } \\
\text { positive }(n=13)\end{array}$ & $\begin{array}{c}\text { Triple } \\
\text { negative }(n=4)\end{array}$ & $\begin{array}{c}P \text { JAK2 } \\
\text { versus CALR }\end{array}$ \\
\hline Sex & & & & & - \\
\hline Male & 27 & 20 & 7 & 0 & \\
\hline Female & 23 & 12 & 6 & 4 & \\
\hline Age (years) median & 52.9 & 54 & 46.1 & 46.5 & 0.045 \\
\hline Duration of symptoms (months) & 16.2 & 13.2 & 26 & 12.7 & 0.16 \\
\hline Spleen size $(\mathrm{cm})$ & 10.3 & 9.3 & 12.1 & 10.5 & 0.037 \\
\hline Hemoglobin (gm/dl) & 10.2 & 11.0 & 9.5 & 9.0 & 0.51 \\
\hline Total leukocyte count $(/ \mathrm{cm})$ & 18300 & 20545 & 12640 & 22045 & 0.07 \\
\hline Platelet counts $\left(\times 10^{9} / \mathrm{L}\right)$ & 3.13 & 3.74 & 1.97 & 2.08 & 0.2 \\
\hline DIPSS score & 1.9 & 2.0 & 1.3 & 2.7 & 0.0004 \\
\hline Myelofibrosis grade on BM biopsy (reticulin stain) & 2.6 & 2.3 & 3.5 & 2.5 & 0.0006 \\
\hline Thrombosis & $3 / 50$ & $3 / 32$ & $0 / 13$ & $0 / 4$ & - \\
\hline
\end{tabular}

DIPSS=Dynamic international prognostic scoring system, BM=Bone marrow, CALR=Calreticulin, JAK2=Janus kinase 2

Table 2: Comparison of the incidence of mutations in primary myelofibrosis with other studies

\begin{tabular}{|c|c|c|c|c|c|c|c|}
\hline Mutation & Li et $a l .{ }^{[5]}$ & Qiao et al. ${ }^{[6]}$ & $\begin{array}{l}\text { Nangalia } \\
\text { et al. }{ }^{[4]}\end{array}$ & Klampfl et al..$^{[3]}$ & Rumi et al. ${ }^{[7]}$ & $\begin{array}{c}\text { Sazawal } \\
\text { et al. }{ }^{|8|}\end{array}$ & $\begin{array}{c}\text { Current } \\
\text { study }\end{array}$ \\
\hline JAK2 & $50 \%(178 / 357)$ & $60.6 \%(20 / 33)$ & $69 \%(35 / 62)$ & $53.2 \%(108 / 203)$ & $64.7 \%(399 / 617)$ & $56.2 \%(45 / 80)$ & $64 \%(32 / 50)$ \\
\hline MPL & $3 \%(11 / 357)$ & $0 \%(0 / 33)$ & $5 \%(2 / 62)$ & $6.4 \%(13 / 203)$ & $4 \%(25 / 617)$ & - & $2 \%(1 / 50)$ \\
\hline CALR & $21 \%(16 / 357)$ & $12.1 \%(4 / 33)$ & $23 \%(9 / 62)$ & $35.5 \%(72 / 203)$ & $22.7 \%(140 / 617)$ & $11.2 \%(9 / 80)$ & $26 \%(13 / 50)$ \\
\hline Triple negative & $27 \%(96 / 357)$ & $27.5 \%(9 / 33)$ & $3 \%(1 / 62)$ & $4.9 \%(10 / 203)$ & $8.6 \%(53 / 617)$ & $32.6 \%(26 / 80)$ & $8 \%(4 / 50)$ \\
\hline
\end{tabular}

CALR $=$ Calreticulin, JAK2=Janus kinase 2, MPL=Myeloproliferative leukemia 
that in rest of the world. CALR-positive patients have better clinical parameters at presentation and have better prognosis as compared to JAK2-positive patients. Indian patients have different hematological profile compared to western counterparts, and also, regional variation in Indian PMF patients in term of incidence of various mutations and clinical parameters can be suspected. Further larger studies are necessary to confirm abovesaid findings, and also elaborative studies are necessary to implicate the role of mutations in survival and treatment response of these patients.

\section{Financial support and sponsorship}

Nil.

\section{Conflicts of interest}

There are no conflicts of interest.

\section{References}

1. Pietra D, Rumi E, Ferretti VV, Di Buduo CA, Milanesi C, Cavalloni C. Differential clinical effects of different mutation subtypes in CALR-mutant myeloproliferative neoplasms. Leukemia 2016;30:431-8.

2. Kralovics R, Passamonti F, Buser AS, Teo SS, Tiedt R, Passweg JR, et al. A gain-of-function mutation of JAK2 in myeloproliferative disorders. N Engl J Med 2005;352: 1779-90.

3. Klampfl T, Gisslinger H, Harutyunyan AS, Nivarthi H, Rumi E, Milosevic JD, et al. Somatic mutations of calreticulin in myeloproliferative neoplasms. N Engl J Med 2013;369:2379-90.
4. Nangalia J, Massie CE, Baxter EJ, Nice FL, Gundem G, Wedge DC, et al. Somatic CALR mutations in myeloproliferative neoplasms with nonmutated JAK2. N Engl J Med 2013;369:2391-405.

5. Li B, Xu J, Wang J, Gale RP, Xu Z, Cui Y, et al. Calreticulin mutations in Chinese with primary myelofibrosis. Haematologica 2014;99: 1697-700.

6. Qiao C, Sun C, Ouyang Y, Wang JJ, Qian SX, Li JY, et al. Clinical importance of different calreticulin gene mutation types in wild-type JAK2 essential thrombocythemia and myelofibrosis patients. Haematologica 2014;99:e 182-4.

7. Rumi E, Pietra D, Pascutto C, Guglielmelli P, Martínez-Trillos A, Casetti I, et al. Clinical effect of driver mutations of JAK2, CALR, or MPL in primary myelofibrosis. Blood 2014; 124:1062-9.

8. Sazawal S, Singh N, Mahapatra M, Saxena R. Calreticulin mutation profile in Indian patients with primary myelofibrosis. Hematology 2015;20:567-70.

9. Wojtaszewska M, Iwoła M, Lewandowski K. Frequency and molecular characteristics of calreticulin gene (CALR) mutations in patients with JAK2 -negative myeloproliferative neoplasms. Acta Haematol 2015; 133: 193-8.

10. Guo H, Chen X, Tian R, Chang J, Li J, Tan Y, et al. Frequencies, laboratory features, and granulocyte activation in Chinese patients with CALR-mutated myeloproliferative neoplasms. PLoS One 2015; 10:e0138250.

11. Tefferi A, Lasho TL, Tischer A, Wassie EA, Finke CM, Belachew AA, et al. The prognostic advantage of calreticulin mutations in myelofibrosis might be confined to type 1 or type 1-like CALR variants. Blood 2014;124:2465-6.

12. Cabagnols X, Defour JP, Ugo V, lanotto JC, Mossuz P, Mondet J, et al. Differential association of calreticulin type 1 and type 2 mutations with myelofibrosis and essential thrombocytemia: Relevance for disease evolution. Leukemia 2015;29:249-52. 